\title{
ACCRETION PROCESSES IN COSMIC SOURCES: The Atmospheres of Accreting White Dwarfs
}

\author{
E.M. Sion* and P. Godon \\ Department of Astrophysics and Planetary Science, Villanova University, Villanova, PA 19085, \\ USA \\ E-mail: edward.sion@villanova.edu, patrick.godon@villanova.edu
}

\begin{abstract}
A comparison is presented between the physical processes operative in single white dwarfs and the physical processes operative in white dwarfs with donor star companions. The physical properties and accretion physics of the hot accreting components in close interacting binaries is presented. The N/C abundance anomaly seen is $\sim 10 \%$ of cataclysmic variables and the presence of absorption lines due to heavy elements in suprasolar abundance are discussed. A synthetic spectral analysis of the FUSE spectrum of the old nova RR Pic reveals reveals an accretion disk with $i=60^{\circ}, M_{\mathrm{wd}}=1.0 M_{\odot}$ and a high accretion rate of $10^{-8} M_{\odot} / \mathrm{yr}$. New evidence from the FUSE and IUE spectra of the symbiotic system CQ Dra suggest it is a triple system consisting of a red giant and a cataclysmic variable companion. The hot component in the symbiotic system, RW Hya, consists of a white dwarf with a surface temperature of $160,000 \mathrm{~K}$ with $\log (g)=6.5$ and no evidence of an accretion disk.
\end{abstract}

Accretion Processes in Cosmic Sources - APCS2016 -

5-10 September, 2016

Saint Petersburg, Russia

${ }^{*}$ Speaker. 


\section{Introduction}

Isolated white dwarf stars, without any interactions with nearby stellar neighbors or close companions, undergo a simple cooling evolution, gradually radiating the thermal energy of the ions in their degenerate cores. while the atomic (and eventually molecular) opacity of their outer, non-degenerate, surface layers, only 60 to $100 \mathrm{~km}$ thick, regulates their rate of heat loss. This cooling rate, which also depends on core mass, determines the timescale of cooling evolution. Complicating this simple picture are a myriad of ongoing physical processes like gravitational and thermal diffusion, weak winds, radiative levitation, convective mixing and very low rates of accretion from either debris disks or interstellar matter, all of which depend to some degree on the changing temperature regimes through which the WD cools [5].

This gentle, non-explosive picture of white dwarf evolution, unaffected by any significant external gains of mass, energy, or angular momentum, cannot begin to describe the violent, more complex evolution of a white dwarf star with a close companion star. White dwarfs in cataclysmic binaries, whether non-magnetic and accreting via a disk or magnetic and accreting via an accretion column (Polars), are heated and spun up by the accretion of mass and angular momentum, undergo thermonuclear burning and thermonuclear explosions, are continually being injected with metal-rich gas, possibly accrete entirely new envelopes, and in the long term, expand if their core masses are eroded by repeated classical nova explosions or shrink if their core masses increase due to continual accretion buildup. Their accretion rates at least ten orders of magnitude higher than single WDs accreting from debris disks or the ISM. In all of these objects moreover, cooling evolution takes on a different meaning since their evolution proceeds with an external heat source (the boundary layer or accretion column) always shining inward and the weight of accreted material coninually compressing their envelopes.

\section{The Photospheres of Accreting White Dwarfs}

The chemical abundances of white dwarfs in cataclysmic variables have been derived largely from high quality HST and FUSE spectra. The observed metal lines are fitted with rotationally broadened theoretical line profiles. Unfortunately, the number of CV WDs with detailed abundance analyses like VW Hyi has been too small to discern any abundance patterns [11].

The most telling characteristic of the abundances of their accreted atmospheres is sub-solar metallicity values. If the replacement time of the accreted atmosphere is shorter than the diffusion timescales of the accreted ions, then the surface abundances would tend to reflect the composition of the infalling matter from the donor star. There is no reason to expect the secondary mass donor stars to have sub-solar metallicity. Rather, one expects that effects of diffusion are responsible for these abundances. At temperatures below 20,000K, radiative forces levitation of the ions is unimportant. The problem is that in order for accretion-diffusion equilibrium to govern the surface abundances, the accretion rate must be low enough, otherwise, the accretion timescale is too short and the abundances tell us nothing about diffusion. The continually changing and unsettled atmosphere of a CV white dwarf where other forms of mixing and spreading of accreted matter are likely involved, presents a challenging theoretical problem. 


\section{The N/C Abundance Anomaly in CV White Dwarfs}

About $10 \%$ of the CVs reveal a high N/C anomaly where the highly non-solar abundances of $\mathrm{N}$ and $\mathrm{C}$ are inferred qualitatively from the $\mathrm{N} \mathrm{V} / \mathrm{C} \mathrm{IV}$ emission line intensity ratios revealed from their accretion disks or accretion columns of magnetic accretors [2]. The N/C abundance anomaly (strong N V 1240/weak or absent C IV 1550) is the hallmark of CNO processing.

However, there are 5 systems (all dwarf novae) in which suprasolar metal abundances are manifested in the FUV photospheric absorption line spectra of CV White Dwarfs exposed during dwarf nova quiescence. Two of these systems, VW Hyi and U Gem, are also know to have large $\mathrm{N} / \mathrm{C}$ ratios. The derived abundances (relative to solar values) from absorption line profile fitting of the HST spectra of VW Hyi are Al 3, Si 0.3, C 0.3, N 3.0, Al 2.0, P 20, and Mn 50 [13].

HST and FUSE spectra of the exposed white dwarf in the dwarf nova U Gem also exhibits a rich array of absorption features due to metals in both IUE and FUSE spectra [12, 6], including suprasolar abundances of $\mathrm{P}$ and $\mathrm{Al}$. In three SU UMa-type CVs with exposed WDs, BW Scl, SW $\mathrm{UMa}$ and $\mathrm{BC}$ UMa, the detected photospheric features reveal aluminum abundances of $3.0 \pm 0.8$, $1.7 \pm 0.5$, and $2.0 \pm 0.5$, respectively [3].

Does the N/C abundance anomaly arise from (1) a formerly more massive secondary donor star (capable of CNO burning) having been peeled away by mass transfer down to its CNO-processed core, or (2) does it originate in the white dwarf itself due to explosive CNO burning associated with nova explosions or possibly by dredgeup and mixing in consequence of a dwarf nova outburst?

If the N/C anomaly originated in the donor, then the donor had to be more massive than its present value to sustain CNO burning. This could be the case if the CVs with the N/C anomaly are the descendants of the supersoft X-ray binaries, when the formerly more massive donor star in the system undergoes thermal timescale mass transfer at a high rate, thus driving steady thermonuclear burning on the white dwarf at the accretion supply rate.

However, [14] have recently shown that at least a fraction of the CVs that reveal the N/C anomaly, the origin of the CNO processed abundances lies with the white dwarf which subsequently contaminated the secondary donor star with the ejecta of many past repeated nova explosions and are transferring this material back to the white dwarf. The central temperatures of any formerly more massive secondary stars in CVs undergoing hydrostatic CNO burning are far too low to produce these suprasolar abundances of heavy elements.

\subsection{The Old Nova RR Pic}

The old novae RR Pic (Nova Pic 1891) is in (or near) its quiescent stage following its nova explosion and continues to accrete at a high rate in the aftermath of the explosion. It exhibits an FUV continuum that is steeply rising into the FUV as well as absorption lines and emission lines of uncertain origin. RR Pic's FUSE spectrum offers not only higher spectral resolution but also wavelength coverage extending down to the Lyman Limit. The dereddened FUV spectrum has been modeled with our grids of optically thick accretion disks and hot, NLTE white dwarf photospheres.

Our modeling analysis indicates that the hot component in RR Pic is likely to be an accretion disk with mass accretion rate of $10^{-8} M_{\odot} /$ yr. However, the disk cannot produce the observed absorption lines. However, for the WD to be the source of the absorption lines it must be very 
hot $\sim 70,000-80,000 \mathrm{~K}$ with a radius several times its expected size (since the WD in RR Pic is massive, it has a smaller radius).

For accretion disk model fits to the FUSE spectrum, with i $=60$ degrees, $M_{\mathrm{wd}}=0.8 M_{\odot}$ and a high accretion rate of $10^{-8} M_{\odot} / \mathrm{yr}$, we obtain a distance of $427 \mathrm{pc}$, which is within the range of RR Pic's parallax distance. This model has a flux level that is too low in the shorter wavelengths of FUSE. When we took the WD mass to be $1.03 M_{\odot}$, then the fit in the shorter wavelengths is improve and a distance of $506 \mathrm{pc}$ is obtained. This accretion disk fit is shown in Fig.1. In order to fit the absorption lines with the disk model, the inclination of the disk has to be set to $3 \mathrm{deg}$, which is almost a face-on disk, while the inclination of RR Pic is known to be $65 \mathrm{deg}$.

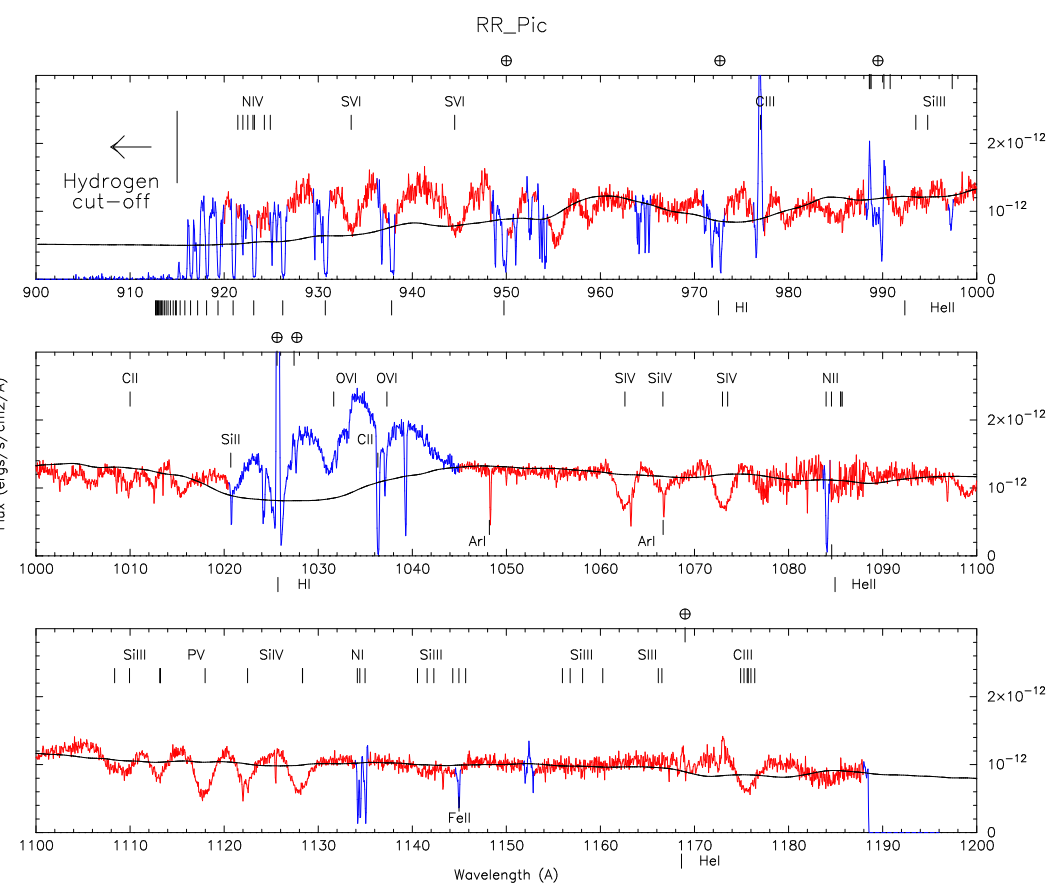

Figure 1: Best Fitting model disk (in black) to the FUSE spectrum of RR Pic (in red). The accretion disk model has an inclination of $\mathrm{i}=60$ degrees, a WD mass $M_{\mathrm{wd}}=1.03 M_{\odot}$ and a high accretion rate of $10^{-8} M_{\odot} / \mathrm{yr}$, giving a distance of 506pc, a result which is with the range of RR Pic's parallax distance. The regions affected by the ISM and broad emission lines have been masked before the fitting and are marked in blue. Note that the absorption lines of the source are not masked as they were initially tentatively modeled with a hot WD model.

Since the FUSE absorption lines do not arise in the disk, (see Fig.1), then they may arise from the white dwarf photosphere. If true, this would imply that we could be seeing significant flux from the post-nova hot white dwarf photosphere itself. However, unless the WD is very hot with an inflated radius, the WD contributes too little flux for this to be a viable possibility.

\subsection{The Symbiotic System CQ Dra}

CQ Dra was originally thought to be a cataclysmic variable in binary orbit with a red giant [8], but was later re-classified as a symbiotic variable. However, as discussed below, the cataclysmic variable interpretation of the hot component may be correct after all. CQ Dra has a reliable HIPPARCOS parallax $\pi=5.25 \pm 0.48$ mas [15]. The orbital period for CQ Dra was determined to be 
1703 days by [1] from radial velocity periodicity searches. This is the longest orbital period of the four systems discussed in this work.

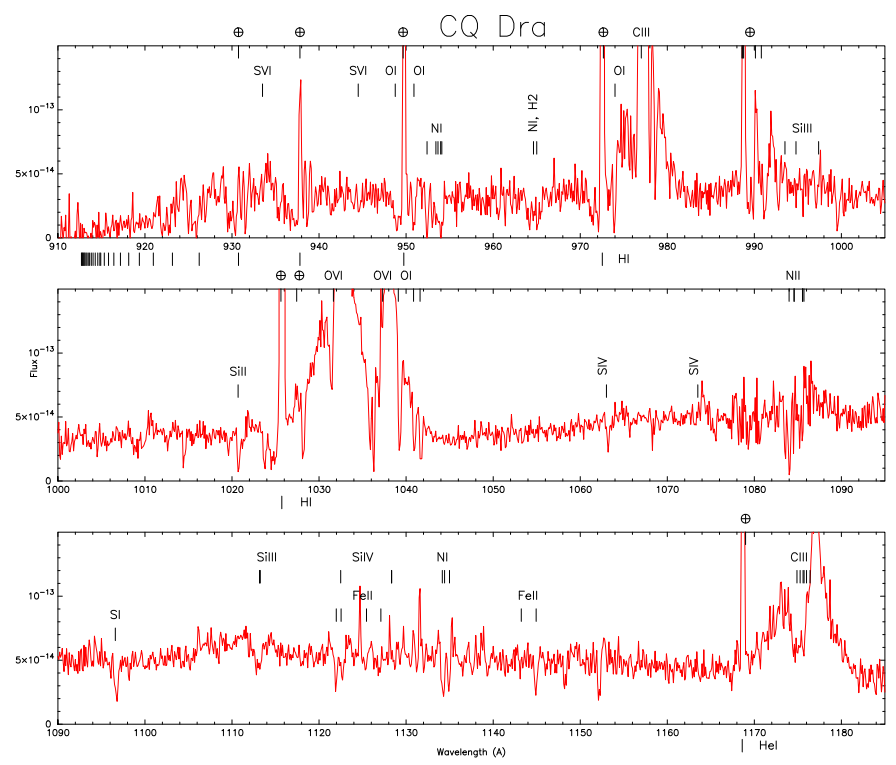

Figure 2: The FUSE spectrum of CQ Dra is shown with line identification. The spectrum has not been dereddened. The spectrum presents little ISM contamination, except possibly for some absorption lines from low ionization species such as Fe II. The source presents broad emission lines from the O VI doublet and the two C'III lines (977 \& 1175) all superposed with sharper absorption lines.

We found a usable FUSE spectrum of CQ Dra in the MAST archive driving our investigation in the following direction. The FUSE spectrum has much lower continuum flux level than the IUE spectra and was obtained in a state of relatively low luminosity.

Curiously the Lyman (series) profiles of $\mathrm{H}$ are not clearly seen, making it more difficult to identify a disk versus a WD.The spectrum does not show signs of interstellar absorption as seen in the FUSE spectra of the other 3 systems in this work, except for atomic H (sharp Lyman series towards the shortest wavelengths). Some of the sharp emission lines are probably due to terrestrial airglow and sunlight reflected in the telescope, and some of the sharp, weak absorptions may also be either terrestrial (e.g. N I 1135) or interstellar (e.g. Fe II 1145).

While identifying lines in the FUSE range via comparison with FUSE spectra of other systems with accreting white dwarfs, we found that the FUSE spectrum of the hot white dwarf in the dwarf nova RU Peg during quiescence, is very similar to the FUSE spectrum of CQ Dra, as seen in Fig.2. There is also a similarity between the IUE spectra (e.g. SWP28355) of the two systems as displayed in Fig.3. At first glance, these similarities would seem to add support to the possibility that the hot component in CQ Dra may indeed be a cataclysmic variable as [8] originally claimed, thus identifying CQ Dra as a triple system. Note however that [16], on the basis of the first X-ray observations of CQ Dra, claim that the hot ultraviolet companion cannot be an AM Her-type, magnetic CV (as argued in [8]) because its X-ray properties are inconsistent with the hot companion's X-ray properties.

The only decent accretion disk fit to the FUSE spectrum of CQ Dra requires ${ }^{\circ}=1 \times 10^{-8} M_{\odot} / y r$ and corresponds to a distance of $\sim 950 \mathrm{pc}$. This fit is shown in Fig.2. The corresponding distance is far beyond the distance of [8]. 


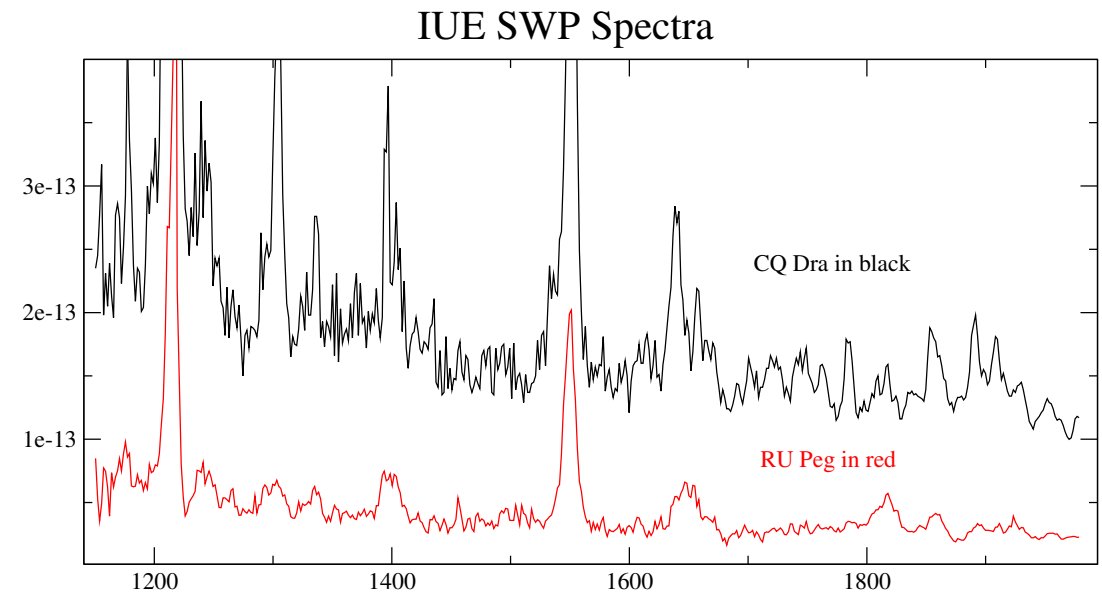

Figure 3: A comparison bwteen the IUE spectrum (SWP28355) of CQ Dra and the IUE spectrum of the white dwarf in the dwarf nova RU Peg during quiescence. For clarity the IUE spectrum of CQ Dra has not been dereddened here. The IUE SWP spectra of CQ Dra in the archive are dominated by strong emission lines of C III (1175), weak N V (1240) emission, weak Si IV (1398) emission, very strong C IV (1550) emission, with He II (1640) being notably absent.

\subsection{The DisK-less System RW Hya}

RW Hydrae (=HD 117970) is an example of an S-type symbiotic system, with the primary classified as a M2 III red giant. The orbital period is 370.2 days [7, 4], and the inclination is quite high, just sufficient for the system to undergo eclipses. Estimates of the white dwarf mass range from 0.3 to $0.6 M_{\odot}$, while that of the red giant is between 0.5 and $2 M_{\odot}[9,4]$. For the distance of the system, we adopt the value based upon corotation of the red giant determined by [9] of $670+/-$ $100 \mathrm{pc}$.

An important feature of RW Hya for white dwarf accretion studies is that there is little or no evidence for an accretion disk. Its multi-wavelength data was analyzed most recently by [10] who dismissed an accretion disk but fit the FUV data with a hot black body SED at $T_{\text {eff }}=10^{5} \mathrm{~K}$.

We carried out single temperature hot WD model fits for a low mass WD to the FUSE spectrum of RW Hya and found a best fit corresponding to a white dwarf with a surface temperature of $160,000 \mathrm{~K}$ with $\log (g)=6.5$. The temperature and gravity are dictated by the shape of the wings of the Lyman series (where possible) and continuum. Assuming a mass of $0.4 M_{\odot}$ and radius of $0.065 R_{\odot}$ yields a scale factor-derived distance of $811 \mathrm{pc}$ confirming that a low mass white dwarf with its larger radius, and a very high temperature gives a distance to RW Hya which is within the error bars of the original Schild et al. distance. This best-fitting NLTE WD model atmosphere fit to the FUSE spectrum is shown in Fig.4. While a low mass (large radius) hot component seems consistent, it cannot be ruled out that the WD is undergoing hydrogen shell burning, has a higher mass but an extended atmosphere whose radius is larger than that given by the WD mass-radius relation. 


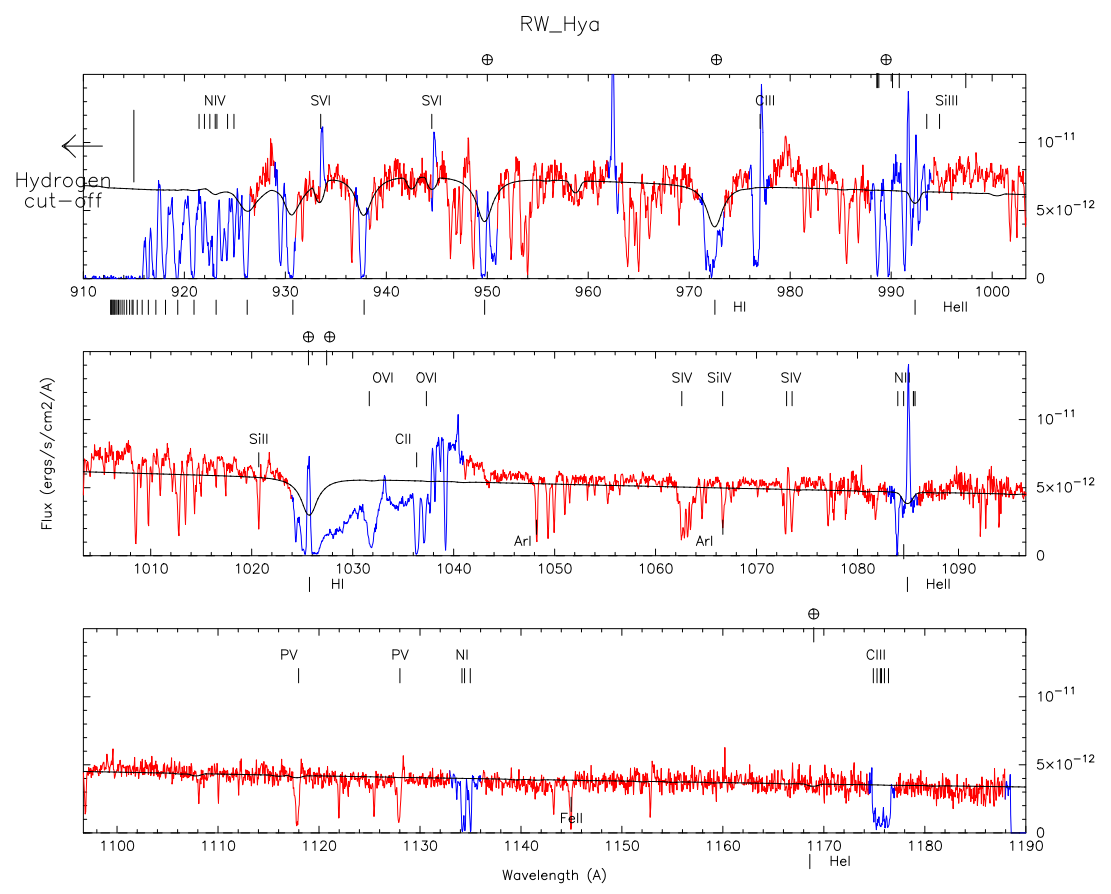

Figure 4: A single temperature hot NLTE WD model fit for a low mass WD to the dereddened FUSE spectrum of RW Hya with a best fit corresponding to a white dwarf with a surface temperature of $160,000 \mathrm{~K}$ and $\log (g)=6.5$. Assuming a mass of $0.4 M_{\odot}$ with a radius of $0.065 R_{\odot}$ yields a scale factor-derived distance of $811 \mathrm{pc}$, confirming that a low mass white dwarf with its larger radius, and a very high temperature gives a distance to RW Hya which is within the error bars of the original Schild et al. distance.

\section{Summary of Conclusions}

- The surface temperatures, chemical abundances, rotation rates and accretion rates onto the hot accreting WD components must be secured in a wide variety of interacting binaries spanning the range of orbital periods from hundreds of days (Symbiotics) down to orbital periods of minutes (AM CVn Systems).

— $\sim 10 \%$ or more of CVs reveal the N/C abundance anomaly.

- Our evidence suggests that CQ Dra really is a triple system consisting of a red giant and a cataclysmic variable.

- The suprasolar abundances of metals with $A>20$ must originate in the white dwarf but either the large N/C in the mass transferring donor originates from peeling down to the $\mathrm{CNO}$ processed core of a formerly more massive secondary OR the large $\mathrm{N} / \mathrm{C}$ as well as the $\mathrm{Al}$ and $\mathrm{P}$ are the result of contamination of the secondary by the products of explosive CNO burning during the "common envelope" stage of a nova outburst.

- A synthetic spectral analysis of the FUSE spectrum of the old nova RR Pic reveals reveals an accretion disk with i $=60$ degrees, $M_{\mathrm{wd}}=1.0 M_{\odot}$ and a high accretion rate of $10^{-8} M_{\odot} / \mathrm{yr}$.

- New evidence from the FUSE and IUE spectra of the symbiotic system CQ Dra suggest it may well be a triple system consisting of a red giant and a cataclysmic variable companion (see however [16]).

- The hot component in the symbiotic system, RW Hya, consists of a white dwarf with a surface temperature of $160,000 \mathrm{~K}$ with $\log (g)=6.5$ and no evidence of an accretion disk. 


\section{Acknowledgments}

This work is supported by NASA Grants NNX13AF12G and NNX13AF11G to Villanova University

\section{References}

[1] P.P.Eggleton, C.D.Bailyn, C.A.Tout, 1989, ApJ, 345, 489

[2] B.T.Gänsicke, et al. 2003, ApJ, 594, 443

[3] B.T.Gänsicke, et al. 2005, ApJ, 629, 451

[4] S.Kenyon, J.Mikolajewska, 1995, AJ, 110, 391

[5] D.Koester, Planets, Stars and Stellar Systems Vol. 4, by T.D.Oswalt, M.A.Barstow, Springer, Dordrecht 2013

[6] K.S.Long, et al.2006, ApJ, 648, 541

[7] P.Merrill, 1950, ApJ, 111, 484

[8] D.Reimers, R.F.Griffith, R.F., A.Brown, 1988, A\&A, 193, 180

[9] Schild et al. 1996, A\&A, 306, 477

[10] A.Skopal, 2005, A\&A, 440, 995

[11] E.M.Sion, et al. 1997, ApJ, 480, L17

[12] E.M.Sion, et al. 1998, ApJ, 496, 449

[13] E.M.Sion, et al. 2001, ApJ, 561, L127

[14] E.M.Sion, W.M.Sparks, 2014, ApJ, 796, 10

[15] L.Van Leeuwen, 2007, A\&A, 474, 653

[16] P. Wheatley, et al. 2003, MNRAS, 346,855 